\title{
Artificial intelligence in musculoskeletal oncological radiology
}

\author{
Matjaz Vogrin ${ }^{1,2}$, Teodor Trojner ${ }^{1}$, Robi Kelc ${ }^{1,2}$ \\ ${ }^{1}$ Department of Orthopaedic Surgery, University Medical Center Maribor, Slovenia \\ ${ }^{2}$ Faculty of Medicine, University of Maribor, Slovenia \\ Radiol Oncol 2021; 55(1): 1-6. \\ Received 1 June 2020 \\ Accepted 29 September 2020 \\ Correspondence to: Assist. Prof. Robi Kelc, M.D., Ph.D., Department of Orthopaedic Surgery, University Medical Centre Maribor, \\ Ljubljanska ulica 5; SI-2000 Maribor. E-mail: robi.kelc @gmail.com \\ Disclosure: No potential conflicts of interest were disclosed.
}

Background. Due to the rarity of primary bone tumors, precise radiologic diagnosis often requires an experienced musculoskeletal radiologist. In order to make the diagnosis more precise and to prevent the overlooking of potentially dangerous conditions, artificial intelligence has been continuously incorporated into medical practice in recent decades. This paper reviews some of the most promising systems developed, including those for diagnosis of primary and secondary bone tumors, breast, lung and colon neoplasms.

Conclusions. Although there is still a shortage of long-term studies confirming its benefits, there is probably a considerable potential for further development of computer-based expert systems aiming at a more efficient diagnosis of bone and soft tissue tumors.

Key words: artificial intelligence; deep learning; tumor recognition; cancer imaging; image segmentation

\section{Introduction}

Primary bone and soft tissue neoplasms present a minority among neoplastic lesions. Due to their rarity, precise radiologic diagnosis often requires an experienced radiologist with special interests in musculoskeletal oncology. To surmount the challenge of making precise diagnoses, and more importantly, to prevent overlooking potentially fatal conditions, attempts to incorporate artificial intelligence and its related techniques into medical practice have occurred in the last decades (Figure 1).

Being first introduced by McCarthy in the 1950s, artificial intelligence (AI) is a general term that describes computer machines that imitate human intelligence. ${ }^{1}$ Machine learning, a subset of AI, uses computational algorithms, which learn with experience and therefore improve the performance of tasks. ${ }^{2}$ The rapid progress of computational power and big data availability allowed the emergence of an even more specialized subfield of machine learning, called deep learning. It is a promising method capable of processing raw data to perform detection or classification tasks. ${ }^{3}$ Deep learning algorithms, implemented as artificial neural networks, mimic biological nervous systems. ${ }^{4}$ The network is organized in layers composed of interconnected nodes imitating architecture in a biologic brain. ${ }^{2,5}$ Nodes are weighted individually for the purpose of increasing data extraction. In order to classify data, weights are automatically and dynamically optimized during the training phase. ${ }^{5,6}$ Regarding the layers, three different kinds are present in each neural network. It begins with an input layer, which receives input data, followed by numerous hidden layers extracting the pattern within the data. It terminates with the output layer, which produces results or output data (Figure 2). ${ }^{5}$

Among the different types of artificial neural networks, convolutional neural networks, in particular, gained attention in radiology due to their high performance in recognizing images. ${ }^{7}$ By calculating the intensity of each pixel or voxel, together with evaluating complex patterns in each image, they provide reliable quantitative image 


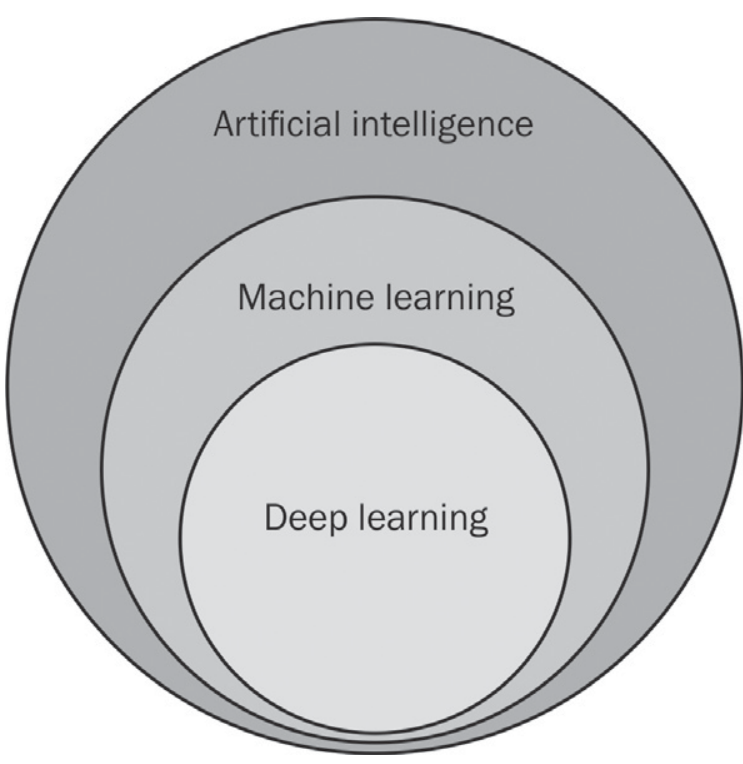

FIGURE 1. Schematic illustration of the hierarchy of artificial intelligence and its machine learning and deep learning subfields.

interpretations, eventually surpassing human performance. 8

However, to build an intelligent machine a training phase is required, which requires sufficient computational power and large datasets. The latter is obtained from radiological images, which is in the domain of radiomics. Radiomics is a process of quantitative extraction of a high number of semantic and agnostic features from diagnostic images. ${ }^{9}$ Its approaches, like feature extraction and feature engineering techniques, are essential in the formation of AI applications. ${ }^{10}$

\section{Artificial intelligence in cancer imaging (oncologic radiology)}

Until recently, radiologists' decisions were based predominantly on his or her experience of recognizing patterns and appreciating various features of each tumor including size, location, intensity, and surface characteristics, all combined with patients' demographic data. However, after many consecutive image interpretations, they were consciously or subconsciously faced with fatigue ${ }^{11}$, which could lead to errors and potentially jeopardize patients' health and own credibility. ${ }^{12}$

AI's potentials are developing exponentially. Instead of qualitative and subjective image interpretations, it allows quantifiable and objective data extraction with the ability to reproduce the same results. ${ }^{13}$ Furthermore, by quantifying information otherwise not detectable to humans, AI may complement clinical decision-making. 5,13

Computer-aided detection (CADe) and computer-aided diagnosis (CADx) algorithms have been used for the last two decades ${ }^{14}$ predominantly in mammography ${ }^{15}$, detection of lung ${ }^{16}$, and colon ${ }^{17}$ malignancies. In contrast to CAD algorithms, which only highlight the features they have been exactly trained for, actual AI systems continue to learn and improve in time. By focusing on the specific diagnosis, systems learn to discover new typical patterns that have not been linked with the disease before. ${ }^{18}$ For example, Beck et al. developed a machine learning-based computer program named "Computational Pathologist (C-Path)" to automatically analyze breast cancer and predict its prognosis. ${ }^{19}$ Importantly, regardless of all well-known histological characteristics that were implemented into the program, C-Path recognized surrounding stroma as another important prognostic factor. ${ }^{19}$ Convolutional neural networks have also given optimistic results in automated detection. ${ }^{6}$ It has been used for automated detection of liver tumors on CT scans with high detection accuracy and precision of $93 \%$ and $67 \%$, respectively. ${ }^{20}$ Similarly, a deep learning-based CADe for detection of brain metastasis on magnetic resonance imaging (MRI) has been developed and achieved a sensitivity of $96 \%$ and reasonable specificity. ${ }^{21}$

In general, characterization is composed of segmentation, diagnosis, and staging of tumors. ${ }^{13}$ Image segmentation is the process used in cancer imaging to outline pathological area and distinguish it from non-pathological adjacent tissue. It can range from planar measurements to advanced 3-dimensional assessment of tumor volume. ${ }^{13}$ Tumors have traditionally been manually labelled by radiologists, which is indeed time-consuming, as well as a subject of interobserver variability. ${ }^{13,22}$ Thus, implementation of AI into automated image segmentation could potentially take over, increase the quality and reproducibility of measurements, and also save time. ${ }^{5,13}$ For example, machine learning has been used for breast density segmentation on mammography, which turns out to be as accurate as manual ones. ${ }^{23}$ Ye et al. successfully proposed and verified a fully automatic nasopharyngeal carcinoma segmentation method based on dual-sequence MRI and convolutional neural network. $^{24}$ The mean dice similarity coefficient (DSC) of the models with only T1 sequence, only T2 sequence, and dual sequence were $0.620 \pm 0.0642$, 


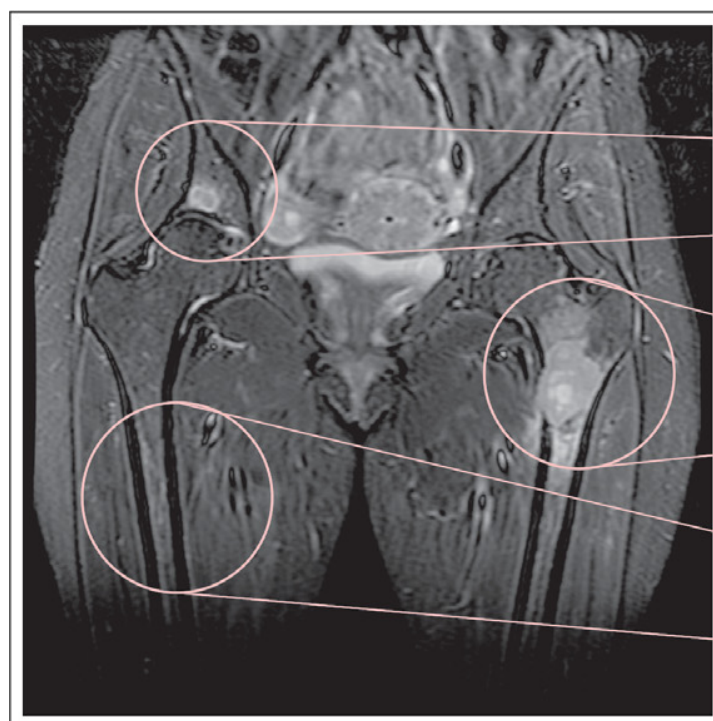

Defined regions of interest

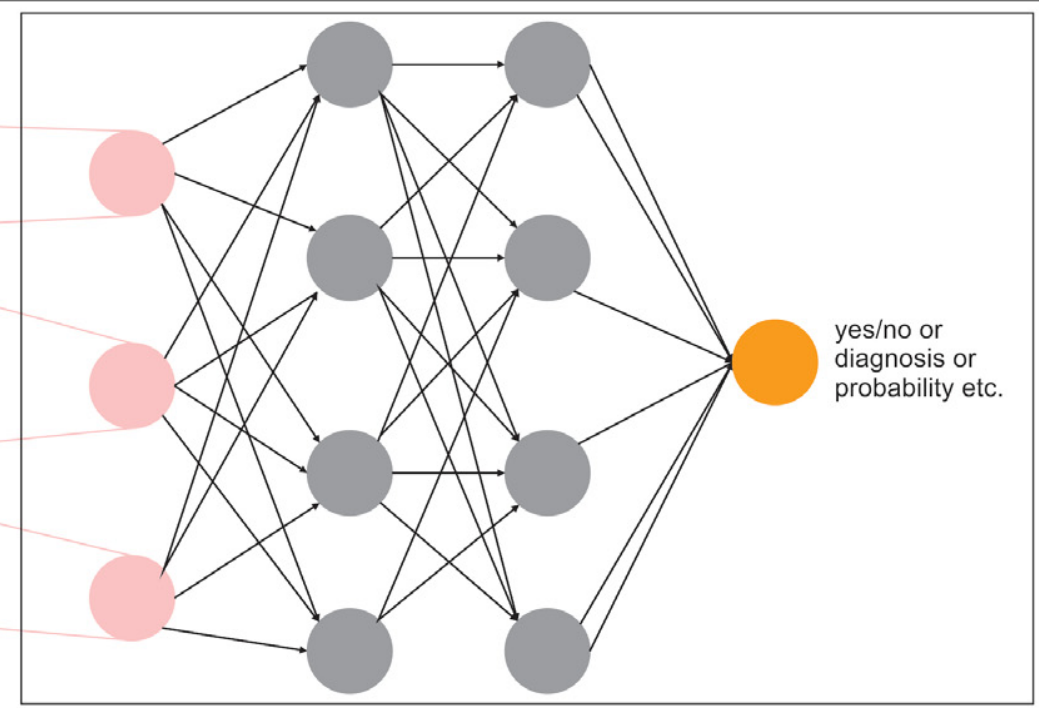

Feature extraction through neural network

FIGURE 2. Schematic presentation of a neural network. Regions of interests (ROI) are defined, either by user or by an automated computer process. These present the input cells (in pink) a neural network. For each ROI the neural network extracts and compute features within the hidden layers (in grey) by using pre-trained data sets. Finally, the output cell offers the final results in different possible forms (yes/no, final diagnosis, probability of malignancy etc.).

$0.642 \pm 0.118$, and $0.721 \pm 0.036$, respectively. The combination of different features acquired from T1 and $\mathrm{T} 2$ sequences significantly improved the segmentation accuracy. ${ }^{24}$

Ability to quantitatively extract tumor features has great potential in the process of making diagnosis. With machine learning, Liu et al. quantitatively represented radiological traits characteristics of lung nodules and showed improved accuracy of cancer diagnosis in pulmonary nodules. ${ }^{25}$ Convolutional neural network has also shown to be an effective and objective method that provides an accurate diagnosis of pancreatic cancer. ${ }^{26}$

Another important aspect of tumor characterization includes staging. Mainly, TNM classification is used to assess the extent of primary tumor, lymph nodes, and metastases and therefore classify the lesion in a specific stage. However, attempts to extend the TNM cancer staging system have been made. For instance, CAD has shown to be a promising method of evaluation of tumor extent and multifocality in invasive breast cancer patients and therefore expanding the staging algorithms. ${ }^{27}$

In tumor response monitoring, many AI approaches have shown some potential. For example, $\mathrm{AI}$ and machine learning have been successfully implemented into the pre-procedural prediction of trans-arterial chemoembolization treatment outcomes in patients with hepatocellular carcinoma using clinical and imaging features. ${ }^{28} \mathrm{Ha}$ et al. demonstrated promising results in the utilization of convolutional neural network to predict neoadjuvant chemotherapy response prior to the first cycle of therapy in breast carcinoma using baseline MRI tumor datasets. ${ }^{29}$ Positive response to chemotherapy led to decreased tumor metabolism, opening a potential opportunity to detect lower accumulation rates of a radiotracer. ${ }^{30}$ Differentiating between responders and non-responders based on low-dose ${ }^{18}$ F-FDG PET/MRI scans might, therefore, be another opportunity of the implementation of convolutional neural networks. ${ }^{6}$

Complementary to radiologic diagnosis, additional advanced methods have been proposed, promising even further advances in cancer management. Liquid biopsy based on circular tumor DNA (ctDNA) analysis may importantly improve early tumor detection, diagnosis, monitoring therapy, and progression in time. ${ }^{31}$ Contrary to standard tissue biopsies, liquid biopsies taken from blood may provide us with detailed biochemical characteristics of the neoplastic lesion and detect potential metastasis. ${ }^{32}$ What is more, a combination of liquid biopsies and radiomics, supervised by deep learning may significantly improve cancer management in the future. 


\section{Artificial intelligence in skeletal tumors}

The first attempts to introduce computational power into diagnostic procedures of primary bone tumors date back to the 1960s. ${ }^{33}$ Based on Bayes' formula, a computer program accurately predicted a bone tumor diagnosis in $77.9 \%$ of cases. Later in 1980 , the same author set a milestone by publishing an article about computed-based radiographic grading of bone tumor destruction. ${ }^{34}$ This was a cornerstone for further research and implementation of neural networks into the diagnosis of focal bone lesions. ${ }^{35,36}$

A scarce number of articles regarding AI and primary bone tumors have been published so far, while considerably more has been done on the detection and segmentation of bone metastasis. For example, Burns et al. successfully identified and segmented sclerotic lesions in the thoracolumbar spine using CADe techniques. The sensitivity for lesions detection was 79\%. ${ }^{37}$ What is more, Wang et al. developed a Siamese convolutional neural network to research the potential of automated spinal metastasis detection in MRI. The proposed approach accurately detected all spinal metastatic lesions with a false-positive rate of 0.4 per case. ${ }^{38}$ Another research proposed a machine learningbased whole-body automatic disease classification tool to distinguish benign processes and malignant bone lesions in ${ }^{18} \mathrm{~F}-\mathrm{NaF}$ PET/CT images. ${ }^{39}$

Healthy and tumorous bone differs in numerous characteristics. Unlike healthy osseous tissue, which consists of cortical and trabecular part, primary bone malignancies may penetrate cortex and spread into adjacent soft tissue, as well as cause swelling around the bone or even weaken the bone architecture and lead to pathological fracture. ${ }^{40}$ Radiologically, they differ in absorption rate, which can be quantitatively evaluated. For example, CADx has been used to detect and classify primary bone tumors into benign and malignant lesions using x-ray images. In their study, Ping et al. an overall greater intensity of pixels for malignant bone tumors compared to benign bone tumors. ${ }^{41}$ Another study by Bandyopadhyay et al. proposed a CADx method to automatically analyze bone $x$ ray images. By integrating several classifiers, the method achieved accurate decisions regarding a bone-destruction pattern, stage, and grade of cancer in $85 \%$ of cases. ${ }^{42}$

When describing sarcomas, diagnosed on MRI, features like tumor size, shape, and enhancement pattern are estimated and taken into consideration along with patient's demographic data. ${ }^{2}$ Machine learning and artificial neural network excel in quantifying and extracting supplementary features, which can correlate with clinical characteristics, diagnosis, and outcomes. Most of these are out of human visual perception and include intervoxel relationships, image intensity analysis, and filtered images analysis. ${ }^{43}$ Deep learning-based algorithm has also been developed to predict survival rates in patients with synovial sarcoma. ${ }^{44}$ Its prediction was more precise compared to the Cox proportional hazard model, which is a commonly used regression model in medical research.

In primary bone tumors, bone tumor matrix, its density, and zone of transition represent suitable characteristics than may be classified through deep learning techniques. In fact, recurrent convolutional neural network outshined experienced musculoskeletal radiologists in bone tumor matrix classification with $86 \%$ vs. $72 \%$, respectively. ${ }^{45} \mathrm{Li}$ et al. proposed a super label guided convolutional neural network to classify CT images of bone tumors. ${ }^{46}$ In comparison, results exceeded the classic convolutional neural network. However, the classification included only nine types of the most common skeletal tumors.

\section{Limitations and future directions}

There are indeed some limitations of AI. First, it could potentially still be a subject of interobserver variability, due to different algorithms used in a neural networks of different AI systems or unequal learning stages in which the systems process a specific task. Standardization is mandatory to establish a large database. Data also needs to be accessible in order to integrate them into large sets. Prior to that quality check, labelling, classification, and segmentation need to be done manually by experts, making the process expensive and time-consuming. ${ }^{13}$ However, introducing deep learning-based techniques to the extensive quality ground-truth training datasets is essential for the development of accurate algorithms. ${ }^{47,48}$ Also, ethical dilemmas should be taken into consideration. When dealing with systems that operate with enormous amounts of data, patients' privacy as well as human dignity may be jeopardized, unless meticulous safety mechanisms are implemented. There are also no long-term follow-up studies available thus far. On the contrary, the appreciating results 
of the already published studies and the presentation of a commercially available application is only a matter of time.

Undoubtedly, all kinds of artificial intelligence are persistently being integrated into the complex management of musculoskeletal tumors and tumors of other sites. Deep learning-based techniques are expected to minimalize false positive rates as well as assure accurate decisions and diagnoses. ${ }^{49}$ Further automatization of radiological tasks is expected to take place in the future. Among physicians, radiologists in particular are required to perform many time-consuming tasks, like image segmentation, delineation of regions of interest, and image annotation. AI techniques have an enormous potential to transform their workflow, which will allow them to focus on more meaningful tasks. ${ }^{11}$

On the other hand, "imaging is not an isolated measure of disease."13 Neoplastic lesions are complex conditions, following DNA mutations that cause abnormal cellular proliferation..$^{50}$ Despite many mutations being discovered and related to specific malignancies, intertumoural and intratumoural heterogeneity exist. ${ }^{51}$ Undoubtedly, molecular approaches, like genetic biomarkers and molecular imaging have already significantly contributed to a better understanding of cancer management. Finally, combining radiomics with other aspects of a broad family of "-omics", including genomics, proteomics, and metabolomics, and therefore drastically expanding datasets available for advanced AI modalities, might move us closer to the precision medicine. ${ }^{52}$

\section{References}

1. Han X-G, Tian W. Artificial intelligence in orthopedic surgery: current state and future perspective. Chin Med J (Engl) 2019; 132: 2521-3. doi: 10.1097/ CM9.0000000000000479

2. Gyftopoulos S, Lin D, Knoll F, Doshi AM, Rodrigues TC, Recht MP. Artificial intelligence in musculoskeletal imaging: current status and future directions. AJR Am J Roentgenol 2019; 213: 506-13. doi: 10.2214/AJR.19.21117

3. LeCun Y, Bengio Y, Hinton G. Deep learning. Nature 2015; 521: 436-44. doi: 10.1038 /nature14539

4. King BF. Guest editorial: discovery and artificial intelligence. $A R J A m J$ Roentgenol 2017; 209: 1189-90. doi: 10.2214/AJR.17.19178

5. Pesapane F, Codari M, Sardanelli F. Artificial intelligence in medical imaging: threat or opportunity? Radiologists again at the forefront of innovation in medicine. Eur Radiol Exp 2018; 2: 35. doi: 10.1186/s41747-018-0061-6

6. Daldrup-Link H. Artificial intelligence applications for pediatric oncology imaging. Pediatr Radiol 2019; 49: 1384-90. doi: 10.1007/s00247-019-04360-1

7. Yasaka K, Abe O. Deep learning and artificial intelligence in radiology: current applications and future directions. PLoS Med 2018; 15: e1002707. doi: 10.1371/journal.pmed.1002707
8. Hosny A, Parmar C, Quackenbush J, Schwartz LH, Aerts HJWL. Artificial intelligence in radiology. Nat Rev Cancer 2018; 18: 500-10. doi: 10.1038/ s41568-018-0016-5

9. Gillies RJ, Kinahan PE, Hricak H. Radiomics: images are more than pictures, they are data. Radiology 2016; 278: 563-77. doi: 10.1148/radiol.2015151169

10. Koçak B, Durmaz EŞ, Ateş E, Kılıçkesmez Ö. Radiomics with artificial intelligence: a practical guide for beginners. Diagn Interv Radiol 2019; 25: 485-95. doi: 10.5152/dir.2019.19321

11. Hirschmann A, Cyriac J, Stieltjes B, Kober T, Richiardi J, Omoumi P. Artificial intelligence in musculoskeletal imaging: review of current literature, challenges, and trends. Semin Musculoskelet Radiol 2019; 23: 304-11. doi: 10.1055/s-0039-1684024

12. Bruno MA, Walker EA, Abujudeh $\mathrm{HH}$. Understanding and confronting our mistakes: the epidemiology of error in radiology and strategies for error reduction. RadioGraphics 2015; 35: 1668-76. doi: 10.1148/rg.2015150023

13. Bi WL, Hosny A, Schabath MB, Giger ML, Birkbak NJ, Mehrtash A, et al. Artificial intelligence in cancer imaging: clinical challenges and applications. CA Cancer J Clin 2019; 69: 127-57. doi: 10.3322/caac.21552

14. Takahashi R, Kajikawa Y. Computer-aided diagnosis: a survey with bibliometric analysis. Int J Med Inform 2017; 101: 58-67. doi: 10.1016/j.ijmedinf.2017.02.004

15. Katzen J, Dodelzon K. A review of computer aided detection in mammography. Clin Imaging 2018; 52: 305-9. doi: 10.1016/j.clinimag.2018.08.014

16. Al Mohammad B, Brennan PC, Mello-Thoms C. A review of lung cancer screening and the role of computer-aided detection. Clin Radiol 2017; 72: 433-42. doi: 10.1016/j.crad.2017.01.002

17. Regge D, Halligan S. CAD: How it works, how to use it, performance. Eur J Radiol 2013; 82: 1171-6. doi: 10.1016/j.ejrad.2012.04.022

18. Kohli M, Prevedello LM, Filice RW, Geis JR. Implementing machine learning in radiology practice and research. AJR Am J Roentgenol 2017; 208: 754-60. doi: 10.2214/AJR.16.17224

19. Beck AH, Sangoi AR, Leung S, Marinelli RJ, Nielsen TO, van de Vijver MJ, et al. Systematic analysis of breast cancer morphology uncovers stromal features associated with survival. Sci Transl Med 2011; 3: 108ra113. doi: 10.1126/ scitranslmed.3002564

20. Afifi A, Nakaguchi T. Unsupervised detection of liver lesions in CT images. Annu Conf IEEE Eng Med Biol Soc 2015; 2015: 2411-4. doi: 10.1109/ EMBC.2015.7318880

21. Zhang M, Young GS, Chen H, Li J, Qin L, McFaline-Figueroa JR, et al. Deeplearning detection of cancer metastases to the brain on MRI. J Magn Reson Imaging 2020: 52: 1227-36. doi: 10.1002/jmri.27129

22. Li XA, Tai A, Arthur DW, Buchholz TA, MacDonald S, Marks LB, et al. Variability of target and normal structure delineation for breast-cancer radiotherapy: a RTOG multi-institutional and multi-observer study. Int $J$ Radiat Oncol Biol Phys 2009; 73: 944-51. doi: 10.1016/j.jijobp.2008.10.034

23. Kallenberg M, Petersen K, Nielsen M, Ng AY, Diao P, Igel C, et al. Unsupervised deep learning applied to breast density segmentation and mammographic risk scoring. IEEE Trans Med Imaging 2016; 35: 1322-31. doi: 10.1109/ TMI.2016.2532122

24. Ye Y, Cai Z, Huang B, He Y, Zeng P, Zou G, et al. Fully-automated segmentation of nasopharyngeal carcinoma on dual-sequence MRI using convolutional neural networks. Front Oncol 2020; in press. doi: 10.3389/fonc.2020.00166

25. Liu Y, Balagurunathan Y, Atwater T, Antic S, Li Q, Walker RC, et al. Radiological image traits predictive of cancer status in pulmonary nodules. Clin Cancer Res 2017; 23: 1442-9. doi: 10.1158/1078-0432.CCR-15-3102

26. Liu SL, Li S, Guo YT, Zhou YP, Zhang ZD, Li S, et al. Establishment and application of an artificial intelligence diagnosis system for pancreatic cancer with a faster region-based convolutional neural network. Chin Med J (Engl) 2019; 132: 2795-2803. doi: 10.1097/CM9.0000000000000544

27. Song SE, Seo BK, Cho KR, Woo OH, Son GS, Kim C, et al. Computer-aided detection (CAD) system for breast MRI in assessment of local tumor extent, nodal status, and multifocality of invasive breast cancers: preliminary study. Cancer Imaging 2015; 15: 1. doi: 10.1186/s40644-015-0036-2 
28. Abajian A, Murali N, Savic L, Laage-Gaupp FM, Nezami N, Duncan JS, et al. Predicting treatment response to intra-arterial therapies of hepatocellular carcinoma using supervised machine learning - an artificial intelligence concept. J Vasc Interv Radiol 2018; 29: 850-57.e1. doi: 10.1016/j. jvir.2018.01.769

29. Ha R, Chin C, Karcich J, Liu MZ, Chang P, Mutasa S, et al. Prior to initiation of chemotherapy, can we predict breast tumor response? Deep learning convolutional neural networks approach using a breast MRI tumor dataset. J Digit Imaging 2019; 32: 693-701. doi: 10.1007/s10278-018-0144-1

30. Kleis M, Daldrup-Link H, Matthay K, Goldsby R, Lu Y, Schuster T, et al. Diagnostic value of PET/CT for the staging and restaging of pediatric tumors. Eur J Nucl Med Mol Imaging 2009; 36: 23-36. doi: 10.1007/s00259-0080911-1

31. Cheng F, Su L, Qian C. Circulating tumor DNA: a promising biomarker in the liquid biopsy of cancer. Oncotarget 2016; 7: 48832-41. doi: 10.18632/ oncotarget. 9453

32. Mader S, Pantel K. Liquid biopsy: current status and future perspectives. Oncol Res Treat 2017; 40: 404-8. doi: 10.1159/000478018

33. Lodwick GS, Haun CL, Smith WE, Keller RF, Robertson ED. Computer diagnosis of primary bone tumors. Radiology 1963; 80: 273-5. doi: $10.1148 / 80.2 .273$

34. Lodwick GS, Wilson AJ, Farrell C, Virtama P, Dittrich F. Determining growth rates of focal lesions of bone from radiographs. Radiology 1980; 134: 577 83. doi: 10.1148/radiology.134.3.6928321

35. Reinus WR, Wilson AJ, Kalman B, Kwasny S. Diagnosis of focal bone lesions using neural networks. Invest Radiol 1994; 29: 606-11. doi: 10.1097/00004424-199406000-00002

36. Piraino DW, Amartur SC, Richmond BJ, Schils JP, Thome JM, Belhobek GH et al. Application of an artificial neural network in radiographic diagnosis. $J$ Digit Imaging 1991; 4: 226. doi: 10.1007/BF03173904

37. Burns JE, Yao J, Wiese TS, Muñoz HE, Jones EC, Summers RM. Automated detection of sclerotic metastases in the thoracolumbar spine at CT. Radiology 2013; 268: 69-78. doi: 10.1148/radiol.13121351

38. Wang J, Fang Z, Lang N, Yuan $\mathrm{H}$, Su M-Y, Baldi P. A multi-resolution approach for spinal metastasis detection using deep Siamese neural networks. Comput Biol Med 2017; 84: 137-46. doi: 10.1016/j.compbiomed 2017.03.024

39. Perk T, Bradshaw T, Chen S, Im H, Cho S, Perlman S, et al. Automated classification of benign and malignant lesions in 18F-NaF PET/CT images using machine learning. Phys Med Biol 2018; 63: 225019. doi: 10.1088/1361$6560 /$ aaebd0

40. Costelloe CM, Madewell JE. Radiography in the initial diagnosis of primary bone tumors. AJR Am J Roentgenol 2013; 200: 3-7. doi: 10.2214/ AJR.12.8488

41. Ping YY, Yin CW, Kok LP. Computer aided bone tumor detection and classification using $x$-ray images. In: Abu Osman NA, Ibrahim F, Wan Abas WAB, Abdul Rahman HS, Ting $\mathrm{H}-\mathrm{N}$, editors. IFMBE proceedings. 4th Kuala Lumpur International Conference on Biomedical Engineering 2008. Berlin, Heidelberg: Springer; 2008. p. 544-7. doi: 10.1007/978-3-540-69139-6_136

42. Bandyopadhyay O, Biswas A, Bhattacharya BB. Bone-cancer assessment and destruction pattern analysis in long-bone x-ray image. J Digit Imaging 2019; 32: $300-13$ doi: $10.1007 /$ s10278-018-0145-0

43. McBee MP, Awan OA, Colucci AT, Ghobadi CW, Kadom N, Kansagra AP, et al. Deep learning in radiology. Acad Radiol 2018; 25: 1472-80. doi: 10.1016/j. acra.2018.02.018

44. Han I, Kim JH, Park H, Kim H-S, Seo SW. Deep learning approach for survival prediction for patients with synovial sarcoma. Tumour Biol 2018; 40 1010428318799264. doi: 10.1177/1010428318799264

45. Reicher JJ, Palo Alto VA, Do BH, Nguyen M, Beaulieu CF. Single-input bone tumor diagnosis based on convolutional neural network classification of bone tumor matrix. SIIM 2018 Annual Meeting, May 31-June 2, National Harbor, MD, 2018

46. Li Y, Zhou W, Lv G, Luo G, Zhu Y, Liu J. Classification of bone tumor on CT images using deep convolutional neural network. In: Kůrková $V$, Manolopoulos Y, Hammer B, lliadis L, Maglogiannis I, editors. Lecture notes in computer science. Artificial neural networks and machine learning - ICANN 2018. Cham: Springer International Publishing; 2018. p. 127-36. doi: 10.1007/978 3-030-01421-6_13
47. Choy G, Khalilzadeh O, Michalski M, Do S, Samir AE, Pianykh OS, et al. Current applications and future impact of machine learning in radiology. Radiology 2018; 288: 318-28. doi: 10.1148/radiol.2018171820

48. Gorelik N, Chong J, Lin DJ. Pattern recognition in musculoskeletal imaging using artificial intelligence. Semin Musculoskelet Radiol 2020; 24: 38-49. doi: 10.1055/s-0039-3400266

49. Burns JE, Yao J, Summers RM. Artificial intelligence in musculoskeletal imaging: a paradigm shift. J Bone Miner Res 2020; 35: 28-35. doi: 10.1002/ jbmr.3849

50. Jokar N, Velez E, Shooli H, Dadgar H, Sadathosseini S, Assadi M, et al. Advanced modalities of molecular imaging in precision medicine for musculoskeletal malignancies. World J Nucl Med 2019; 18: 345. doi: 10.4103/ wjnm.WJNM_119_18

51. Hinohara K, Polyak K. Intratumoral heterogeneity: more than just mutations. Trends Cell Biol 2019; 29: 569-79. doi: 10.1016/j.tcb.2019.03.003

52. Acharya UR, Hagiwara Y, Sudarshan VK, Chan WY, Ng KH. Towards precision medicine: from quantitative imaging to radiomics. J Zhejiang Univ Sci B 2018; 19: 6-24. doi: 10.1631/jzus.B1700260 